\title{
Usos y potencialidad de la Higuerilla (Ricinus communis) en sistemas agroforestales en Colombia
}

\author{
$\underline{\text { María I. Sánchez S. }}{ }^{1}, \underline{\text { Román D. Castañeda } S}{ }^{2 *}$, Marlon J. Castañeda S. $^{3}$ \\ ${ }^{1}$ MVZ Joven Investigador Colciencias 2014 - Universidad del Tolima. Santa Helena. Parte alta. Ibagué - \\ Tolima. \\ ${ }^{2}$ Profesor asistente - Universidad del Tolima. \\ ${ }^{3}$ Profesor Ocasional - Programa de Ingenería Forestal - Universidad de Santander - UIS - Sede Malaga. \\ Grupo de Investigación Sistemas Agroforestales Pecuarios \\ *Autor para correspondencia Email: rcastaneda@ut.edu.co
}

\begin{abstract}
RESUMEN. La higuerilla (Ricinus communis) es una planta oleaginosa cultivada en todas las regiones tropicales, con especial adaptación a zonas semiáridas. En los últimos años su cultivo ha aumentado en regiones como Brasil, China e India, países en los cuales se ha investigado para mejorar las condiciones agronómicas a fin de aumentar su productividad. En Colombia su cultivo se ha fomentado en vista a la demanda nacional e internacional del aceite de ricino, ya que posee múltiples usos y características particulares que lo convierten en un producto versátil y de alta calidad. El objetivo de esta revisión es plantear el uso de higuerilla como un componente de un sistema agroforestal, en el cual se realicen asociaciones con gramíneas y especies animales, para de esta manera obtener una mayor eficiencia económica por hectárea, al producir aceite de ricino, carne, leche, crías, huevos, entre otros productos, además de recuperar el suelo y conservar la biodiversidad del ecosistema.
\end{abstract}

Palabras clave: aceite de ricino, biodiesel, sistemas agroforestales alternativos

\section{Uses and potential of Castor (Ricinus communis) in agroforestry systems in Colombia}

\begin{abstract}
The castor oil plant is an oilseed cultivated in all the tropical regions, with special adaptation to semi-arid areas in the last few years has increased its cultivation in regions such as Brazil and India countries that have been investigated to improve the agronomic conditions in order to increase yields cultivation in Colombia has been promoted in view of the national and international demand of the castor oil, which is generated from the multiple uses that has this and its derivatives, due to the particular features of that has this quality. The aim of this review is propose the use of castor plant as a component of an agroforestry system, in which associations with grasses and animal species are made, to obtain greater economic efficiency per hectare, the produce castor oil, meat, milk, breed, eggs, among other products, in addition to recovering the soil and conserve the biodiversity of the ecosystem.
\end{abstract}

Keywords: alternative agroforestry systems, biodiesel, castor oil

\section{Introducción}

La planta de higuerilla se caracteriza por su rusticidad y adaptabilidad a diversas condicionestopográficas y climatológicas, crece comúnmente en regiones áridas, con suelos arenosos y es conocida por la población rural como una maleza sin significancia económica. Sin embargo debido a las características específicas de su aceite se ha perfilado como cultivo promisorio generador de materia prima de alta calidad para diversas industrias a nivel mundial (Franco, 1999).

Según estudios realizados por el Instituto de biocombustibles, energías alternativas y renovables en la Universidad de Pamplona (Norte de Santander) en 2006, el aceite de higuerilla o aceite de ricino, es uno de los que genera mayor rendimiento de peso en relación a la semilla, 
variando entre el $51 \%$ y $55 \%$. Según SoleraSteller et al., (2015) la conversión de aceite en biodiésel, casi duplica el obtenido para la soya. Además, la calidad de este aceite es destacada por ser soluble en alcohol y poseer alta viscosidad y densidad en la naturaleza, lo que le da propiedades únicas para algunas industrias especializadas (Jiménez, 2010).

Datos reportados por (Vega, 2010), sugieren que la producción de semilla para variedades mejoradas en el país es de 3 a 4.5 ton/ha/año (dependiendo de la densidad de siembra), correspondiente a 1.5 a 2 ton/ha/año de aceite y a su vez éste origina $687 \mathrm{gal} / \mathrm{ha} / \mathrm{año}$ de biodiesel; empleando a 0.64 personas por hectárea para su cultivo y transformación

En los últimos años la investigación de las oleaginosas y en especial la planta de higuerilla ha tenido un desarrollo importante en países como Brasil y Colombia en la que ha tomado parte el sector público y privado, enfocado esfuerzos en desarrollar variedades con características específicas para algunas zonas, con mejores rendimientos agronómicos, ofreciendo al mercado semilla certificada, que garantiza producción y calidad de producto, además se pueden ver otras posibilidades de encadenar su cultivo con otros sistemas productivos.

A nivel mundial el aceite de higuerilla representó el $0,0078 \%$ de la producción total de aceites vegetales (Córdoba, 2012). En el 2009, la producción mundial de higuerilla fue de 1.473.751 ha con una producción total de 1.499,111 ton de semilla (Tabela 1); Los países productores más importantes son: India con 840.000 hectáreas, China 210.000 hectáreas, Brasil 159.205 hectáreas, Paraguay 11.000 hectáreas y una producción de semilla de 1.098.000 toneladas, 190.000 toneladas, 90.384 toneladas, 13.000 toneladas, respectivamente. Así mismo, en los últimos 25 años, se ha evidenciado un aumento del consumo de aceite de higuerilla, al pasar de 400.000 toneladas de aceite en 1985, a 610.000 toneladas de aceite, en el 2010 (FAO, 2010).

Tabla 1. Producción y valores de la Higuerilla en el 2009.

\begin{tabular}{lllll}
\hline Hectáreas Sembradas & Semilla producida & \$ USS Semilla/ton* & Aceite higuerilla & \$ USS Aceite/ton* \\
\hline 1.473 .751 ton & 1.499 .111 ton & 1.400 & 610.000 ton & 2.700 \\
\hline
\end{tabular}

Fuente: Rico et al., (2011) y Córdoba (2012). *Valores internacionales.

En cuanto al precio del aceite, éste es influenciado por los otros productos agrícolas, especialmente, los aceites vegetales; por lo que, el precio de referencia para el aceite de higuerilla fue un $66 \%$ superior al de soya, desde el 2003 hasta el 2011. Éste ha fluctuado desde US\$ 650 la tonelada en febrero de 2002, a un máximo de US\$ 2.700 tonelada en febrero de 2011 (Severino et al., 2012).

Según Severino \& Godin (2005), en Colombia se está fomentando el cultivo de especies oleaginosas no comestibles como, Higuerilla, Jatropha y Sacha Inchi, como materia prima para la industria del biodiesel, farmacéutica, cosmetología y de fertilizantes. Empresas como Higueroil, Colombiana de biocombustibles S.A., Industria Colombiana de Ricino y Productos Derivados - INCORP S.A.S. de Antioquia, la Federación Nacional de biocombustibles en Cundinamarca y Agronegocios de Colombia en Nariño, cuentan con planta procesadora de aceite y tienen como misión promocionar el encadenamiento productivo y comercial de oleaginosas para la extracción, elaboración y venta de aceites vegetales y biocombustibles de segunda generación, garantizando al productor el adecuado manejo del cultivo y la compra de la cosecha, inclusive encadenando con la financiación de proyectos por medio del Banco Agrario.

El objetivo de esta revisión es conocer los usos actuales del aceite de ricino y sus subproductos en la industria, explorar el potencial de mercado de la semilla y aceite con creciente demanda y proponer su uso en sistemas agroforestales pecuarios.

\section{Usos del aceite de Ricino en la Industria}

El aceite de higuerilla no es comestible, es ampliamente empleado como una materia prima para muchos productos de la industria química, debido a la presencia en su estructura de grupos hidroxilos y dobles enlaces, haciéndolo adecuado para muchas reacciones químicas $\mathrm{y}$ modificaciones (Ogunniyi, 2006). 
Por sus características químicas, es más versátil que otros aceites, lo que permite ser usado como precursor de diferentes productos de la industria química.

En la actualidad el aceite de higuerilla es usado en diversas industrias entre las que se encuentran: la industria de plásticos. Según, Jaramillo et al., (2007), este aceite tiene potencial para ser utilizado como materia prima hidrocarbonada en la obtención de polímeros como poliuretano, poliamidas, resinas epoxicas entre otros. El aceite de ricino tiene la ventaja de ser generado a partir de una planta de cultivo anual, quees competitiva en costos y además no compite con la seguridad alimentaria al no ser comestible, tal como ocurre en la obtención de estos productos a partir de aceite de palma o de colza. En la Industria de lubricantes, Pérez et al., (2009), realizaron pruebas para mejorar el rendimiento de los motores al utilizar lubricantes minerales y mezclarlos con aceite de higuerilla, en busca de favorecer la adherencia y la untuosidad a superficies metálicas para protección de los motores (2500-6000 rpm), observando un comportamiento estable a diferentes porcentajes de manejo, pero sin ofrecer una protección adicional debido a la baja velocidad de fricción, lo que indica que el potencial de uso del aceite de higuerilla se encuentra en motores de altas revoluciones sometidos a temperaturas ambientales bajas, como por ejemplo en la industria aeronáutica. EL aceite de ricino también es utilizado en la industria cosmética y farmacéutica, para la fabricación de labiales, tónicos capilares, champús, brillos, emulsificantes, desodorantes, ceras, anticaspa, laxante, purgantes, catártico y emoliente; al igual que en la industria de pinturas, tintes y para el procesamiento de textiles (Córdoba, 2012).

\section{Aceite de ricino como insumo del biodiesel}

El biodiesel es considerado como un combustible renovable, biodegradable y medioambientalmente limpio, que puede convertirse de forma gradual y satisfactoria en un substituto del diésel mineral. Además, el biodiesel no contiene azufre y no es corrosivo (Durán et al., 2009).

El biodiesel originado del aceite de ricino posee una mayor acción lubricante, en comparación a los demás lubricantes producidos a partir de otras materias primas, pudiendo promover en general una mayor vida útil a los motores. Los procedimientos necesarios de preparación de la materia prima para la conversión en biodiesel, tiene por objetivo hacer más efectivo la reacción de transesterificación para conseguir la máxima tasa de conversión (Duran et al, 2009).

En Argentina se elaboró un biocombustible a partir de aceite de ricino que tiene gran potencial en la industria aeronautica. Según pruebas realizadas en la Base Antártica Marambio de la fuerza aérea Argentina durante la temporada de invierno y verano. El valor agregado de esta innovación a diferencia de los combustibles vegetales ya existentes, es la resistencia al frío, el alto punto de niebla y la temperatura de cristalización están entre los parámetros no deseados de la mayoría de los biodiesel a ser usados, ya que el descenso de temperatura provocado por la altura exige que el combustible aeronáutico soporte temperaturas cercanas a $50^{\circ} \mathrm{C}$, en el caso del biodiesel aparentemente supera las pruebas (Mellincovsky, 2012).

\section{Uso potencial de subproductos de la higuerilla en la alimentación animal}

\section{Usos de las hojas y tallos}

Las hojas y tallos de la planta de higuerilla aunque no es apta para consumo, por su contenido toxico de ricina y ricinina, tiene una amplia variedad de usos desde la fabricación de papel o papiros con finalidad artesanal en México (Escoto Garcia, 2010), hasta para alimentación de gusanos Eri productores de seda artesanal (Montoya, 2010).

\section{Torta de higuerilla}

Abono: El residuo de la extracción del aceite, es la llamada torta que corresponde aproximadamente al 50\% del peso de la semilla. La presencia de factores antinutricionales (ricina y ricinina) no permite la utilización de la torta como alimento para animales. Las tortas de aceite no comestibles como es el caso de higuerilla y algunas variedades de jatropha, se utilizan como fertilizantes orgánicos (Duarte, 2008). Esta tenía un valor en el mercado como fertilizante de $\$ 60$ USS/Ton, para el 2011. (Sayegh \& Cardenas, 2011).

Alimentacion animal: La torta de ricino tiene un alto valor nutricional por ser rica en proteínas, fibras, materia mineral y grasa. El alto contenido de proteínas también la hace una buena materia 
prima para la producción de aminoácidos, tales como arginina, cistina, fenilalanina, leucina, histidina, isoleucina, lisina, metionina, tirosina, treonina, triptófano y valina (Duarte, 2008).

Severino (2005), reporto que el contenido de aminoácidos esenciales (lisina y triptófano) es mucho más pequeño en la torta de ricino que en torta de soja, limitando de esta manera el uso de la semilla de ricino como fuente de proteína en la alimentación de animales monogástricos (caballo, cerdo, aves, pescado). Por el contrario, puede ser buena fuente de nutrientes para los rumiantes, ya que la mayoría de la proteína utilizada para estos animales proviene de proteína microbiana sintetizada en el rumen, la torta se puede utilizar como fuente de proteína en las dietas bajas en aminoácidos esenciales.

En vista de la calidad nutricional de la torta, Furtado etal., (2012), realizaron un experimento en donde se detoxifico torta de higuerilla, evaluando piedra Caliza $60 \mathrm{~g}, 10 \mathrm{~g}$ de urea, fosfato monodicalcico $60 \mathrm{~g}$, colocándola en autoclave por 15 minutos a 60/psi para promover la desaparición completa de la ricina en la torta de ricino, obteniendo que este proceso no mejoró el consumo y la digestibilidad de nutrientes.

La torta de semilla de ricino sin tratamiento de desintoxicación se puede utilizar en la alimentación de las ovejas como un alimento rico en proteína, participando en hasta un $8 \%$ de la ración total, sin causar una reducción significativa en el consumo y digestibilidad. Entre tanto, de acuerdo con Duarte (2008), La composición bromatológica de la torta de higuerilla detoxificada es: materia seca $97,26 \%$; proteína $41,07 \%$, fibra cruda $37,49 \%$, extracto etéreo $1,34 \%$, cenizas $4,3 \%$, calcio $0,35 \%$, fosforo $0,43 \%$.

\section{Potencial de asociación del cultivo de higuerilla en sistemas agroforestales}

Los sistemas agroforestales son el conjunto de técnicas de uso y manejo dela tierra que implica la combinación de árboles forestales con cultivos agrícolas (anuales/perennes), con animales o con ambos a la vez, en una parcela, ya sea simultáneamente o sucesivamente, para obtener ventajas de la combinación. (Chavez, 2007). El objetivo de los SAF (Sistemas Agroforestales) es optimizar la producción total por área, para mejorar la economía familiar del productor y su calidad de vida, ademas de ser una produccion diversificada y mas duradera (sustentable). La clasificacion de los sistemas agroforestales toma en cuenta los componentes que los conforman y la distribucion que tienen estos en el tiempo y en el espacio.

Se reconoce como interacciones la manera en que los diferentes componentes de un sistema se relacionan entre sí. La integración de árboles cultivos y animales resultan en interacciones positivas y negativas. Dentro los factores positivos tenemos la eficiencia en la utilización de la energía solar, supresión de malezas, reducción de la velocidad del viento, conservación de la humedad del suelo, mejoramiento de la productividad del suelo, bienestar animal traducido en mejores parámetros zootécnicos, entre otras; dentro los factores negativos tenemos la competencia por luz, agua, espacio, nutrientes, entre otras. Es por esto que el balance entre las interacciones positivas y negativas depende de las especies sembradas, su arreglo espacial, su densidad y manejo (Limongui, 2002).

La acción de la competencia de las plantas, ya sea de diferentes especies o de la misma, reducen la producción de la planta. Sin embargo, Rico et al (2011), midiendo la cantidad de semilla en $\mathrm{kg} / \mathrm{ha}$ no observaron diferencias significativas entre las densidades de 10.000 y 5.000 plantas por ha. Lo que indica que no necesariamente se deben tener cultivos con altas densidades para obtener una buena productividad y un beneficio económico (Figura 1).

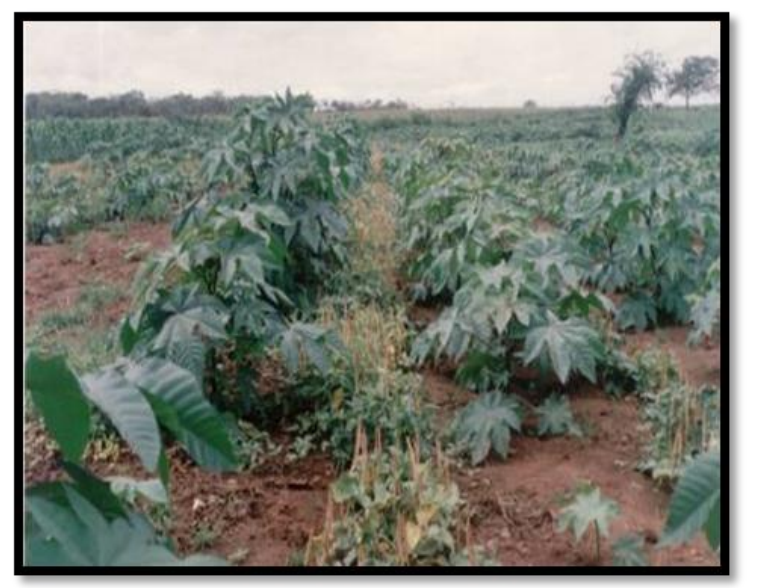

Figura 1. Cultivo higuerilla (Ricinus communis) asociado con frijol. Tomado de Cavalcante, 2004.

Otro aspecto por analizar es la opción de plantar la higuerilla como componente agrícola en un sistema agroforestal, sin embargo, existe la limitante de la toxicidad de la planta, la cual eventualmente podría ser consumida por los 
animales, pero tal como lo plantea Duarte (2008) y Anderssona (2012) los animales adquieren inmunidad a dosis bajas de la ricina, además animales con disponibilidad de alimento no consumen la planta tal como demostró Anderssona (2012), en un ensayo con Jartropha (planta toxica), en asociación con gramíneas pastoreadas por cabras en Sudáfrica, donde se planteó la Jartropha como especie promisoria para sistemas pastoriles, ya que hubo un bajo a nulo consumo de Jartropha por parte de las cabras, que se dedicaron al consumo de la gramínea disponible.

De esta manera seria interesante realizar estudios de investigación donde se evalué la asociación de la higuerilla con gramíneas, ya que existe un potencial económico y ambiental en ese tipo de sistemas.

\section{Conclusiones}

El cultivo de higuerilla es una opción promisoria para Colombia ya que posee suelos y condiciones medio ambientales aptas para su siembra y además favorece la inclusión social, empresarial y la sustentabilidad del sector agropecuario. Por otro lado, existen empresas que brindan la asesoría técnica y garantizan la compra del producto, además para las empresas transformadoras es un mercado potencial a nivel mundial, en vista de sus características especiales lo hacen una materia prima de excelente calidad para la producción de biodiesel, la industria cosmética, farmacéutica, lubricantes, pinturas, entre otras. Entre tanto, es necesario evaluar el potencial de la higuerilla en sistemas agroforestales pecuarios, ya que en la actualidad no existen estudios acerca de este tema, lo cual puede convertirse en una alternativa sustentable para las familias del sector rural.

\section{Referencias Bibliográficas}

Anderssona, E. F; Eversona, T. \& Everson, C. S. (2012). Management of oil producing Jatropha curcas silvopastoral systems: Risk of herbivory by indigenous goats and competition with planted pastures. School of Life Sciences. University of KwaZulu-Natal. Scottsville, Pietermaritzburg. South África.

Cavalcante, F. S. (2004). A Importância da Mamona para a agricultura familiar no estado da Paraíba. Revista electrónica de ciências, 27.
Chavez, D. (2007). Manual de agroforesteria. Proyecto manejo sostenible de recursos naturales. ISBN: 978-99953-65-00-4. San Lorenzo, Paraguay.

Córdoba, O. J. (2012). Comportamiento eco fisiológico de variedades de higuerilla (Ricinus communis L.) para la producción sostenible de aceite y biodiesel en diferentes agroecosistemas colombianos. Universidad Nacional de Colombia. Facultad de Ciencias Agrarias. Medellín, Colombia.

Duarte, M. J., Delmondes, M. A. \& Severino, L. S. (2008). Utilização de coprodutos da mamona na alimentação animal, III. Congreso Brasilero de Mamona. Energía y ricinoquimica. Salvador.

Durán, J. M., Retamal, N. \& Moratiel, R. (2009). El cultivo de rícino (ricinus communis 1.) En Andalucía: una alternativa para producción de biodiesel. Cultivos Energéticos Alternativos. España.

Escoto Garcia, T. (2010). Taller para el aprovechamiento de la Higuerilla y Tule en la elaboración de amate y Papiro en Amatitan. Departamento de Madera Celulosa y Papel. Centro universitario de ciencias exactas e ingenierías. Universidad de Guadalajara. México.

Franco, A. J. (1999). Higuerilla: de maleza a la industria. Periódico el Tiempo. Colombia. dispobible

en

http://www.eltiempo.com/archivo/documento/ MAM-861270.

Furtado, R. N., Carneiro, M. S. S. \& Candide, M. J. D. (2012). Valor nutritivo de las dietas que contienen semillas de ricino sometido a métodos alternativos de desintoxicación para las ovejas. Ciencia y tecnología de los animales y la inspección de productos de origen animal. Arquivo Brasileiro de Medicina Veterinária e Zootecnia, 64.

Jaramillo, F., Pilonieta, A. M. G., \& Riaño, L. J. C. (2007). Poliuretanos degradables a partir de aceite de higuerilla. Scientia et Technica, 4 $36,1-6$.

Jiménez, M. A., Gallegos, C. H. \& Níñez-Colín, C. A. (2010). Relación entre variables climáticas con la morfología y contenido de aceite de semillas de higuerilla (Ricinus communis 1.) De Chiapas. Universidad Autónoma Chapingo. México. 
Limongui, A. J. \& Andrade, J. R. F. (2002). Caracterización del sistema agroforestal "maíz con árboles dispersos" en la cuenca del rio Carrizal, Manabí, Ecuador. Turrialba, Costa Rica.

Mellincovsky, A. (2012). LBio-Jet, biocombustible a partir de aceite no comestible para aviones. Aceso en 8 de octubre de 2015, disponible en http://www.elotromate.com/ciencia/bio-jetbiocombustible-a-partir-de-aceite-nocomestible-para-aviones/.

Montoya, J. L. (2010). Cria del gusano Eri, para la obtención de seda artesanal. Delegacion de SAGARPA S.L.P. México.

Ogunniyi, D. S. (2006). Castor oil: A vital industrial raw material. En: Bioresource Technology.

Pérez Ruiz, E., Garrido, J. \& Graciano, N. (2009). Comportamiento de la temperatura de trabajo de un lubricante mineral utilizando aceite de ricino como aditivo. Scientia et Technica Año XV, No 43, Universidad Tecnológica de Pereira.

Rico, H. R., Tapia, L. M. \& Teniente, R. (2011). Guía para cultivar higuerilla (Ricinus communis L.) en Michoacán. Investigación regional del pacífico centro campo experimental valle de Apatzingán Apatzingán. Folleto Técnico, 1.

Sayegh, O. A. \& Cardenas, A. L. (2011). Plan de empresa basado en el uso de aceite de higuerilla para la industria cosmética. Facultad de Ciencias Administrativas y Económicas. Maestría en administración. Universidad ICESI. Cali, Colombia.
Severino S., L., Cordoba, G., O J. Zanotto, M. D. \& Auld, D. L. (2012). The influence of the caruncle on the germination of castor seed under high salinity or low soil water content. Seed Science and Technology, 40, 139-143.

Severino, L. S. \& Souza de Gondim, T. M. (2005). Curso sobre el cultivo de higuerilla. EMBRAPA - Brasil. Traducido por: Diaz Quintana, A. \& Silva Acuña, R. INIA Venezuela.

Severino, L. S. (2005). O que sabemos sobre a torta da mamona. Empresa Brasileira de Pesquisa Agropecuária. Brasil.

Solera-Steller, P., Moreira-González, I. \& Hernández-López, J. (2015). Descriptores botánicos para caracterizar germoplasmas de Ricinus communis de diferentes zonas de Costa Rica. Revista Tecnología en Marcha, 28, 37-46.

Vega, O. (2010). Atlas de la agroenergía y los biocombustibles en las Américas: II Biodiesel. Programa hemisférico en agroenergía y biocombustibles. Instituto interamericano de cooperación para la agricultura. San José, Costa Rica.

Recebido em Diciembre 23, 2015

Aceito em Janeiro 18, 2016

License information: This is an open-access article distributed under the terms of the Creative Commons Attribution License, which permits unrestricted use, distribution, and reproduction in any medium, provided the original work is properly cited. 